A Jin Lee, ${ }^{1}$ Nae Ri Kim, ${ }^{1}$ Eun Jung Yang, ${ }^{1}$ Kyeong A So, ${ }^{1}$ Sun Joo Lee, ${ }^{1}$ Ji Young Lee, ${ }^{1}$ Tae Jin Kim, ${ }^{1}$ Soon-Beom Kang, ${ }^{2}$ Seung-Hyuk Shim ${ }^{1, *}$

'Konkuk University School of Medicine, Seoul, Korea

(nastassja@hanmail.net)

${ }^{2}$ Hosan Women Hospital, Seoul, Korea

Objective: To assess the outcomes of retreatment using progestin in patients with recurrent endometrial cancer (EC) after achieving complete response (CR) with fertility sparing hormonal treatment. Methods: We reviewed retrospectively patients who had recurrence after achieving CR by fertility sparing treatment using progestin with presumed stage IA, grade 1, endometrioid EC. All recurrent patients were given medroxyprogesterone acetate or megestrol acetate with concurrent LNG-IUD. The primary end point was the response rate. The secondary end points were pregnancy outcome, pathologic disease progression, and 2 nd recurrence rate.

Results: Of 55 recurrent patients, 50 received progestin retreatment. With median retreatment duration of 8 (3-56) months, the CR rate of the retreatment group was $78 \%$ (39/50); $76.2 \%$ in EC group and $87.5 \%$ in endometrial intraepithelial neoplasia group. Of 11 patients not achieving CR to progestin retreatment, 3 patients underwent surgical treatment and none had extrauterine spread of disease. Of 20 patients who attempted to conceive after achieving CR, 8 patients became pregnant. Of the 39 patients achieving CR after 1st recurrence, 20 (51.2\%) patients had 2nd recurrence with a median follow-up duration of 15 (9-146) months. Of those, 16 patients received second-line progestin retreatment and 11 (68\%) of these patients achieved CR. All patients who underwent retreatment are alive without evidence of disease.

Conclusion: First-line and second-line progestin retreatment in patients with recurrent EC are as effective and safe as initial fertility sparing treatment. Therefore, it may provide an opportunity for young patients who still want to preserve fertility after recurrence.

Oral (OM9)

Endometrial Hyperplasia, Endometrial Intra-epithelial Neoplasia, and Endometrial Cancer

https://doi.org/10.3802/jgo.2021.32.S1.0M9

\section{Indirect Excess Dose Volume Ratio (iRex): a potential toxicity controlling parameter for image-guided adaptive brachytherapy in inoperable endometrial cancer}

Tissana Prasartseree, " Pittaya Dankulchai

Division of Radiation Oncology, Department of Radiology, Faculty of Medicine, Siriraj Hospital, Mahidol University, Bangkok, Thailand (tissana.p@gmail.com)
Objective: Radiation toxicities still occurred despite achievable dose constraints in image-guided adaptive brachytherapy (IGABT) of inoperable endometrial carcinoma. Indirect Excess Dose Volume Ratio (iRex; doi: 10.5114/jcb.2020.100377) was explored for its potential toxicity restriction.

Methods: All inoperable endometrial cancer cases receiving IGABT with/without external beam radiation therapy (EBRT) in 2013-2017 were reviewed for loco-regional control, distant metastasis-free, cancer-specific, and overall survival (OS) rate. iRex60 were genuinely generated for radiation toxicity analysis, in addition to conventional brachytherapy constraints $\left(D_{2 c c}\right)$. Descriptive, Kaplan-Meier, Mann-Whitney U test, and net reclassification index (NRI), were utilized.

Results: Thirteen inoperable endometrial cancer patients (median age 74 years old, follow-up time 43 months) underwent IGABT alone and IGABT+EBRT for 1 and 12 patients. Four-year locoregional control, distant metastasis free survival, causespecific survival were all $100 \%$, but OS was $83.9 \%$. Crude grade $\geq 3$ gastrointestinal (GI) toxicity occurred in $3(23.1 \%)$ patients, yielding accrual 4-year incidence of $15.4 \%$, whereas late-persistent toxicity was presented in only $1(7.7 \%)$ patient. No high-grade genitourinary toxicity occurred. For dosimetric data (12 patients), comparing grade 3-5 with 0-2 late GI toxicity group, mean $\mathrm{D}_{2 \mathrm{cc}}$ rectum were 62.0 (range 51.7-76.1) and 58.7 (52.2-73.3) Gy EQD ${ }_{2,3}(\mathrm{p}=0.78)$, while mean iRex60 were 2.86 $(2.02-4.03)$ and $1.94(1.13-2.96)(\mathrm{p}=0.12)$, respectively. iRex- $\mathrm{D}_{2 c c}$ scatter plot showed lower toxicity incidence underneath iRex cut-point of 2. However, absolute and additive NRI of $-8.3 \%$ $(\mathrm{p}=0.93), 33.3 \%(\mathrm{p}=0.51)$ showed no significant improvement of toxicity prediction with iRex60 incorporation.

Conclusion: iRex60 showed potential cut-point of 2 from iRex$\mathrm{D}_{2 \mathrm{cc}}$ scatter plot, lower iRex60 seems to be associated with lower toxicity incidence, yet not statistically significant. Larger sample is required for validation.

\section{Ovarian cancer}

Oral (001)

Epithelial Ovarian Cancer including Borderline Tumor https://doi.org/10.3802/jgo.2021.32.S1.001

\section{Efficacy and safety of niraparib combined with oral etoposide in platinum-resistant/ refractory ovarian cancer (PROC): a single arm, phase 2 study}

\author{
Jiaxin Yang,,$^{1, *}$ Huimei Zhou, ${ }^{1}$ Qingshui Li, ${ }^{2}$ Depu Zhang, ${ }^{2}$ \\ Dongyan Cao,' Yang Xiang' \\ 'Peking Union Medical College Hospital, Chinese Academy of
}


Medical Sciences and Peking Union Medical College, Beijing, China (Yjiaxin20202002@63.com)

${ }^{2}$ Shandong Cancer Hospital Affiliated to Shandong University, Shandong Academy of Medical Sciences, Jinan, China

Objective: Treatment options for platinum-resistant/refractory ovarian cancer (PROC) are few and efficacies are limited especially for primary platinum-resistant/refractory disease. PARP inhibitor (PARPi) combined with chemotherapy could improve the treatment outcome. So, we designed a phase 2 study to evaluate the efficacy and safety of niraparib combined with oral etoposide in PROC.

Methods: Eligible patients had PROC with histologically confirmed non-mucinous epithelial ovarian, fallopian tube, or primary peritoneal cancer. They must have had 1-2 prior lines of platinum-based chemotherapy, and no prior therapy with PARPi. Patients received niraparib $200 \mathrm{mg}$ or $100 \mathrm{mg}$ alternate once daily and oral etoposide $50 \mathrm{mg}$ on day 1-20 of a 30-day cycle. Oral etoposide was administered for a maximum of 6-8 cycles. Treatment was continued until disease progression, patient withdrawal or unacceptable toxic effects. The primary endpoint is progression free survival evaluated by RECIST v1.1. Results: Recruitment began on 22 May 2020. The 20 patients were enrolled to date. The mean number of prior lines of chemotherapy was 1.3 for these patients, which means almost all of them had primary platinum-resistant/refractory disease. Median follow-up period was 3.2 months (1.1-6.5). One patient achieved a partial response, 6 had stable disease (RECIST v1.1). The 15 patients had discontinued the study treatment due to disease progression or intolerable gastrointestinal toxicity. The 5 patients remained on treatment. No new safety signals were identified.

Conclusion: Niraparib combined with oral etoposide had a manageable toxicity profile and showed promising antitumor activity in PROC with 1-2 prior lines of platinum-based chemotherapy. Study recruitment is ongoing.

Trial Registration: ClinicalTrials.gov Identifier: NCT04217798

Oral (002)

Epithelial Ovarian Cancer including Borderline Tumor

https://doi.org/10.3802/jgo.2021.32.S1.002

\section{Factors predict postoperative morbidity in advanced stage ovarian cancer patients who underwent cytoreductive surgery: a systematic review and meta-analysis}

\footnotetext{
Malika Kengsakul,,", Gatske M. Nieuwenhuyzen-de Boer, ${ }^{1}$ Suwasin Udomkarnjananun, ${ }^{2}$ Stephen J. Kerr, ${ }^{3}$

Christa D. Niehot, ${ }^{4}$ Heleen J. van Beekhuizen'
}

'Department of Gynecologic Oncology, Erasmus MC Cancer Institute, University Medical Center Rotterdam, Rotterdam, The Netherlands (malika@g.swu.ac.th)

${ }^{2}$ Faculty of Medicine, King Chulalongkorn Memorial Hospital, Chulalongkorn University, Bangkok, Thailand

${ }^{3}$ Research Affairs, Faculty of Medicine, Chulalongkorn University, Bangkok, Thailand

${ }^{4}$ Medical Library, Erasmus MC Cancer Institute, University Medical Center Rotterdam, Rotterdam, The Netherlands

Objective: The cornerstone treatment for advanced stage epithelial ovarian cancer (EOC) is cytoreductive surgery followed by adjuvant chemotherapy. An independent prognostic factor for advanced stage EOC is the amount of residual disease following cytoreductive surgery. Recently, more extensive surgery has been performed in order to achieve complete cytoreduction. The aim of this study was to evaluate adjustable predictive factors for postoperative morbidity after extensive cytoreductive surgery for advanced stage EOC.

Methods: The Embase, MEDLINE, Web of Science, Cochrane Library and Google Scholar databases were searched for articles from the database inception to May 2021. Meta-analysis was conducted to determine factors associated with postoperative complication by using random-effects models.

Results: The 1,953 citations were retrieved from searching strategy. Fourteen relevant studies, involving 15,219 ovarian cancer patients, were included in the review. Severe 30day postoperative complication occurred in 2,338 (15.4\%) patients. Postoperative mortality rate was $1.93 \%$. Meta-analysis demonstrated that patient with following risk factors; age $(\mathrm{p}<0.001)$, Eastern Cooperative Oncology Group score $>0$ ( $\mathrm{p}=0.001)$, albumin level $<3.5 \mathrm{~g} / \mathrm{dL}(\mathrm{p}<0.001)$, presence of ascites on computed tomography scan $(\mathrm{p}=0.013)$, stage IV disease $(\mathrm{p}<0.001)$ and extensive surgical procedure $(\mathrm{p}<0.001)$ has a significant increase risk of developing postoperative complication. Surgical procedures including peritonectomy $(\mathrm{p}=0.012)$, splenectomy $(\mathrm{p}<0.001)$ and colon surgery $(\mathrm{p}<0.001)$ were significant predictors for postoperative complication. Conclusion: Our study demonstrated that patient's performance status and hypoalbuminemia were the only significant adjustable preoperative risk factors associated with postoperative complication. Preoperative assessment of serum albumin is essential to identify patients at risk for complication after cytoreductive surgery. A future randomized controlled trial on preoperative nutritional support and physiotherapy prehabilitation should be conducted to evaluate the impact on postoperative complication outcome. 